University of Nebraska - Lincoln

DigitalCommons@University of Nebraska - Lincoln

USDA Wildlife Services - Staff Publications

U.S. Department of Agriculture: Animal and Plant Health Inspection Service

2021

\title{
Powassan Virus Experimental Infections in Three Wild Mammal Species
}

Nicole M. Nemeth

Colorado State University, nmnemeth@uga.edu

J. Jeffrey Root

USDA APHIS Wildlife Services

Airn E. Hartwig

Colorado State University

Richard A. Bowen

Colorado State University

Angela M. Bosco-Lauth

Colorado State University

Follow this and additional works at: https://digitalcommons.unl.edu/icwdm_usdanwrc

Part of the Natural Resources and Conservation Commons, Natural Resources Management and Policy Commons, Other Environmental Sciences Commons, Other Veterinary Medicine Commons, Population Biology Commons, Terrestrial and Aquatic Ecology Commons, Veterinary Infectious Diseases Commons, Veterinary Microbiology and Immunobiology Commons, Veterinary Preventive Medicine, Epidemiology, and Public Health Commons, and the Zoology Commons

Nemeth, Nicole M.; Root, J. Jeffrey; Hartwig, Airn E.; Bowen, Richard A.; and Bosco-Lauth, Angela M., "Powassan Virus Experimental Infections in Three Wild Mammal Species" (2021). USDA Wildlife Services Staff Publications. 2444.

https://digitalcommons.unl.edu/icwdm_usdanwrc/2444

This Article is brought to you for free and open access by the U.S. Department of Agriculture: Animal and Plant Health Inspection Service at DigitalCommons@University of Nebraska - Lincoln. It has been accepted for inclusion in USDA Wildlife Services - Staff Publications by an authorized administrator of DigitalCommons@University of Nebraska - Lincoln. 


\title{
Powassan Virus Experimental Infections in Three Wild Mammal Species
}

\author{
Nicole M. Nemeth, ${ }^{1,2 \star}$ J. Jeffrey Root, ${ }^{3}$ Airn E. Hartwig, ${ }^{4}$ Richard A. Bowen, ${ }^{4}$ and Angela M. Bosco-Lauth ${ }^{4}$ \\ ${ }^{1}$ Department of Population Health, Southeastern Cooperative Wildlife Disease Study, University of Georgia, Athens, Georgia; ${ }^{2}$ Department of \\ Pathology, Southeastern Cooperative Wildlife Disease Study, University of Georgia, Athens, Georgia; ${ }^{3}$ United States Department of Agriculture/ \\ APHIS, National Wildlife Research Center, Fort Collins, Colorado; ${ }^{4}$ Department of Biomedical Sciences, College of Veterinary Medicine and \\ Biomedical Sciences, Colorado State University, Fort Collins, Colorado
}

\begin{abstract}
Powassan virus (POWV) is a tick-borne virus maintained in sylvatic cycles between mammalian wildlife hosts and ticks (primarily Ixodes spp.). There are two currently recognized lineages, POWV-lineage 1 (POWV-L1) and deer tick virus (DTV; lineage 2), both of which can cause fatal neurologic disease in humans. Increased numbers of human case reports in the northeastern and north central United States in recent years have fueled questions into POWV epidemiology. We inoculated three candidate wildlife POWV reservoir hosts, groundhogs (Marmota monax), striped skunks (Mephitis mephitis), and fox squirrels (Sciurus niger), with either POWV-L1 or DTV. Resulting viremia, tissue tropism, and pathology were minimal in most inoculated individuals of all three species, with low (peak titer range, $10^{1.7}-10^{3.3}$ plaqueforming units $/ \mathrm{mL}$ serum) or undetectable viremia titers, lack of detection in tissues except for low titers in spleen, and seroconversion in most individuals by 21 days postinoculation (DPI). Pathology was limited and most commonly consisted of mild inflammation in the brain of POWV-L1- and DTV-inoculated skunks on four and 21 DPI, respectively. These results reveal variation in virulence and host competence among wild mammalian species, and a likely limited duration of host infectiousness to ticks during enzootic transmission cycles. However, POWV can transmit rapidly from tick to host, and tick co-feeding may be an additional transmission mechanism. The rare and low-level detections of viremia in these three, common, wild mammal species suggest that vector-host dynamics should continue to be explored, along with eco-epidemiological aspects of enzootic POWV transmission in different regions and virus lineages.
\end{abstract}

\section{INTRODUCTION}

Powassan virus (POWV; family Flaviviridae; genus Flavivirus) is a tick-borne virus maintained in sylvatic cycles between mammalian wildlife hosts and numerous tick species, primarily Ixodes spp. Human infections with POWV can lead to lifelong neurologic disease and death, and reports of such cases have increased in recent years in parts of the United States. ${ }^{1,2}$ The current understanding of natural virus maintenance and transmission dynamics stems from human case reports, knowledge of tick host feeding behavior, as well as rare POWV detections in wild mammals and ticks, and early experimental infection trials and serologic surveys in wild mammals in enzootic regions. ${ }^{3-6}$ Powassan virus has been detected in at least five tick species in North America, some or all of which may be undergoing geographic range expansion. ${ }^{7}$ The recent characterization of distinct lineages of POWV, that is, lineage 1 (i.e., Powassan prototype; POWV-lineage 1 [POWV-L1]) and lineage 2 (deer tick virus; DTV), has fueled questions into host-vector dynamics within sylvatic transmission cycles for each lineage, and whether public health risk varies between the two lineages. ${ }^{5,8,9}$

With the continued rise in the diagnoses of clinical human cases of POWV-L1 and DTV in North America, addressing knowledge gaps in transmission ecology, including major vertebrate virus-amplifying (i.e., reservoir) hosts, is increasingly urgent. Direct comparisons in candidate reservoir host data are needed, and should include viremia profiles, potential health effects, tissue tropism, and serologic responses for POWV-L1 and DTV, as both pose a demonstrated, zoonotic risk. ${ }^{10}$ Experimental trials in multiple candidate reservoir host species using currently established virological methods would allow for more accurate comparisons among host species and between

*Address correspondence to Nicole M. Nemeth, Southeastern Cooperative Wildlife Disease Study, University of Georgia, 589 D.W. Brooks Dr., Athens, GA 30602. E-mail: nmnemeth@uga.edu virus lineages. Furthermore, climatic conditions that permit expansion of promiscuous tick vectors into regions where competent, naive hosts abound may inadvertently increase risk of human exposures. ${ }^{5}$ Altered climatic patterns, including temperature and precipitation, as well as urbanization, ensure dynamic and complex host-virus-vector relationships and add to the urgency for which an improved understanding of POWVL1 and DTV eco-epidemiology are needed. ${ }^{7}$

The primary objective of the present study was to assess groundhogs (Marmota monax), striped skunks (Mephitis mephitis), and fox squirrels (Sciurus niger) as potential POWV-L1 and DTV reservoir hosts through experimental infections. Specific objectives included 1) characterization of lineage 1 and 2 viremia profiles, tissue tropism during acute infections, and seroconversion rates; 2) comparison of these parameters among the three host species and between the two POWV lineages; and 3) assessment of clinical and pathological effects of POWV-L1 and DTV infections in these three species.

\section{MATERIALS AND METHODS}

Animal sources and husbandry. Adult groundhogs were live-trapped using large tomahawk traps (size 108; Tomahawk Live Trap Co., Tomahawk, WI) in January 2016 in Clay, Onondaga County, New York, and housed indoors in mosquitoproof rooms at Colorado State University (CSU) for 2 years before the study. In January 2018, 19 (nine adults and 10 subadults [i.e., > 9 months old]; 12 females and seven males) groundhogs were transferred to a biosafety level (BSL)-3 containment facility at CSU where they were group-housed in 3.7 wide $\times 5.5$ long $\times 3.7 \mathrm{~m}$ high rooms with four, approximately $0.46 \times 0.30 \times 0.33 \mathrm{~m}$ hutches for cover. While in containment, one of the adult female groundhogs gave birth to 4 groundhogs (hereafter referred to as "juveniles"). Groundhogs were provided fresh water and rabbit chow (Ranch Way Feeds, Ft. Collins, CO) supplemented with raw carrots, sweet potatoes, apples, peanuts, almonds, and pecans. 
Eight fox squirrels (six subadults and two adults; two females and six males) were live-trapped in small tomahawk traps (size 106) in Larimer County, Colorado, in July 2018 and housed in standard animal rooms for approximately 4 weeks before transfer to BSL-3 containment at CSU. Squirrels were individually housed in 0.76 long $\times 0.92$ wide $\times 0.46 \mathrm{~m}$ high cages with closed-end polyvinyl chloride pipes for cover and provided fresh water and mixed nuts (hazelnuts, walnuts, Brazil nuts, pecans, cashews), dry corn kernels, and apples.

Eight, adult, male, captive-reared striped skunks were purchased from Ruby Fur Farms (New Sharon, IA) in July 2018 and housed in outdoor animal pens for approximately 4 weeks before being transferred to BSL-3 containment at CSU. Skunks were subcutaneously injected with ivermectin (Vetrimec $^{\text {TM }} 1 \%$, VETone, Norbrook Laboratories Ltd., Newry Co. Down, Northern Ireland) approximately 1 week before inoculation to reduce parasite burden. Skunks were individually housed in 0.66 wide $\times 1.02$ long $\times 0.76 \mathrm{~m}$ tall cages with the same style hutches as for the groundhogs and were provided fresh water and fresh fruit (e.g., apples, pears, and cantaloupe), dried mealworms, and Mazuri ${ }^{\circledR}$ omnivore diet (Brentwood, MO).

Skunks and squirrels were subcutaneously implanted with Implantable Electronic identification Transponders (Bio Medic Data Systems, Inc., Seaford, DE) before the study for assessment of internal body temperatures and individual identification numbers. All individuals were acclimated to BSL-3 containment facilities for at least 2-3 days before inoculation. Animal rooms underwent natural seasonal light cycles and were maintained at approximately $18-20^{\circ} \mathrm{C}$. Animal care and handling were conducted under institutional animal care approval (CSU and U.S. Department of Agriculture/Animal and Plant Health Inspection Service).

Viruses. Virus strains used for inoculation included DTVSP0 M1V3 June, 92005 and POWV LB September 15, 2007 (for POWV-L1). The DTV strain was originally isolated from an Ixodes scapularis tick in Spooner, Wisconsin, in 2005 and passaged once on baby hamster kidney (BHK) cells and once on Vero cells. The POWV-L1 strain was isolated from a fatal human case in 1958 and passaged once on BHK cells and once on Vero cells.

Inoculation. Animals inoculated with POWV-L1 included four adult, four subadult, and two juvenile ( $\sim 1$ month old) groundhogs; three subadult fox squirrels; and three adult skunks. In addition, four adult and four subadult groundhogs, two adult and two subadult fox squirrels, and four adult skunks were inoculated with DTV. All animals except groundhog juveniles were prescreened for antibodies to POWV and DTV with samples considered seronegative at $<50 \%$ neutralization at a 1:10 serum dilution. Groundhog adults and subadults each received a virus inoculum dose of $\sim 10^{6.0}$ plaque-forming units (PFUs) of POWV-L1 or DTV in $0.5 \mathrm{~mL}$ of BA1 medium injected subcutaneously, equally divided over the left and right inguinal regions. The groundhog juveniles received an intentionally lower dose of $\sim 10^{2.8}$ PFU POWV-L1 in $0.1 \mathrm{~mL}$ of BA1 medium. Skunks and fox squirrels received a virus dose of $\sim 10^{4.9}$ PFU POWV-L1 or $10^{4.0}$ PFU DTV in $0.1 \mathrm{~mL}$ of BA1 medium. One adult, two subadult, and two juvenile ( 1 month old) groundhogs; one subadult fox squirrel; and one adult striped skunk were sham-inoculated with BA1 medium (Hank's M-199 salts, 1\% bovine serum albumin, sodium bicarbonate $[350 \mathrm{mg} / \mathrm{L}]$, penicillin [100 units $/ \mathrm{mL}$ ], streptomycin [100 $\mathrm{mg} / \mathrm{L}]$, and amphotericin $B$ [1 mg/L] in $0.05 \mathrm{M}$ tris, $\mathrm{pH}$ 7.6) and served as negative pathology controls.

Sampling scheme. Groundhogs and skunks were anesthetized for inoculation and blood collection $(0.5 \mathrm{~mL})$ via the jugular vein. Squirrels were anesthetized for inoculation and manually restrained for blood collection $(0.2 \mathrm{~mL})$ via the femoral vein. Anesthesia was injected via intramuscular injection of Zetamine (ketamine $\mathrm{HCl}$; VetOne, Boise, ID, dose $100 \mathrm{mg} /$ $\mathrm{mL}$ ketamine with 1:10 xylazine). Groundhogs received a Zetamine volume of $0.7-0.8 \mathrm{~mL}$, skunks $0.5-0.75 \mathrm{~mL}$, and squirrels $0.2 \mathrm{~mL}$; when necessary, isoflurane gas was used for short-term anesthetic maintenance. Weights (via digital scale to $0.1 \mathrm{~g}$ ) and body temperatures were recorded for skunks and squirrels when handled for inoculation and blood collection. Temperature change was assessed as patterns of average change among inoculated, same-species individuals over time. Observational behavioral and clinical assessments were made twice daily throughout the experiment.

Each species followed one of two experimental time lines (Table 1). The first of these prioritized assessment of pathology in acute infections; these individuals were bled on 2 and 4 days postinoculation (DPI; groundhogs) or 1-4 DPI (skunks and squirrels) and euthanized on four DPI. The second time line prioritized assessment of viremia profiles and seroconversion; individuals were bled on 1, 3, 5, 7, 10, and 21 DPI (groundhogs) or $1-3,5,7,14$, and 21 DPI (skunks and squirrels) and euthanized on $21 \mathrm{DPI}$. One of two POWV-L1-inoculated groundhog juveniles was euthanized and bled on three DPI and the second on $21 \mathrm{DPI}$; blood collection from these juveniles was limited to one occasion, immediately following euthanasia via intracardiac stick. At each sampling time point, blood was either collected into serum separator tubes (skunks and groundhogs; BD Microtainer, Becton-Dickinson, Franklin Lakes, $\mathrm{NJ}$ ) or placed into cryovials and diluted in $0.5 \mathrm{~mL}$ BA1 for an approximate 1:10 serum dilution (squirrels). Blood or diluted blood was allowed to clot for 30-60 minutes at room temperature, centrifuged at $10,000 \times g$ for 3 minutes, and frozen to $-80^{\circ} \mathrm{C}$ until testing.

Animals were euthanized by intracardiac injection of sodium pentobarbital overdose following anesthesia as described earlier. Necropsies were performed within 2 hours of euthanasia. Nutritional condition was determined by the presence and subjective amount of subcutaneous, intra-abdominal, and perirenal adipose deposition. The following tissues were collected, placed in $10 \%$ buffered formalin and processed routinely for microscopic examination, and stained with hematoxylin and eosin: skeletal muscle (quadriceps and biceps), heart, trachea, lung with bronchus, thyroid gland, spleen, liver, gall bladder, kidney, adrenal gland (skunks and fox squirrels only), gonad, pancreas, esophagus, stomach, small intestine, large intestine, lymph node (mesenteric and thoracic), haired skin, adipose, bone marrow (femur), urinary bladder, eye, sciatic nerve, thoracic spinal cord, and brain (cerebrum, cerebellum, and brain stem). Fresh samples of the heart, spleen, kidney, and brain from each animal euthanized on four DPI were individually stored in cryovials and frozen to $-80^{\circ} \mathrm{C}$ for virus isolation. Immediately before assay, approximately $1 \mathrm{~cm}^{3}$ samples of thawed brain, kidney, heart, and spleen were individually homogenized in $1 \mathrm{~mL}$ BA1 medium as previously described. ${ }^{11}$

Virology and serology. Sera and tissue homogenates were serially diluted 10 -fold by Vero cell plaque assay ${ }^{12}$ to 
TABLE 1

Summary of viremia and seroconversion in groundhogs, skunks, and fox squirrels experimentally inoculated with POWV-lineage 1 or DTV

\begin{tabular}{|c|c|c|c|c|c|}
\hline \multirow[b]{2}{*}{ Species } & \multirow[b]{2}{*}{ Virus } & \multirow{2}{*}{$\begin{array}{l}\text { No. viremic/ } \\
\text { no. inoculated }\end{array}$} & \multicolumn{2}{|c|}{ Viremia $(\mathrm{PFU} / \mathrm{mL} \text { serum) })^{\star}$} & Seroconversion (21 DPI) \\
\hline & & & Mean; range peak & DPI & No. seroconverted/no. tested (PRNT 80 range) $\dagger$ \\
\hline \multirow[t]{2}{*}{ Groundhog (Marmota monax) $\ddagger$} & POWV-L1 & $2 / 8$ & $10^{1.7} ; 10^{<1.7}-10^{2.3}$ & $3-4$ & $3 / 4(10-320)$ \\
\hline & DTV & $1 / 8$ & $10^{2.4} ; 10^{<1.7}-10^{3.3}$ & 3 & $3 / 4(80-320)$ \\
\hline \multirow[t]{2}{*}{ Striped skunk (Mephitis mephitis) } & POWV-L1 & $1 / 3$ & $10^{1.8} ; 10^{<1.7}-10^{2.3}$ & $2-3$ & $1 / 1(160)$ \\
\hline & DTV & $0 / 4$ & $<10^{1.7}$ & - & $2 / 2(160-320)$ \\
\hline \multirow[t]{2}{*}{ Fox squirrel (Sciurus niger) } & POWV-L1 & $0 / 3$ & $<10^{1.7}$ & - & $1 / 1(10)$ \\
\hline & DTV & $1 / 4$ & $10^{2.1} ; 10^{<1.7}-10^{2.7}$ & 3 & $2 / 2(40-80)$ \\
\hline
\end{tabular}

DPI = days postinoculation; DTV = deer tick virus; PFU = plaque-forming unit; POWV-L1 = Powassan virus-lineage 1.

${ }^{*}$ Minimum threshold for virus detection: $10^{1.7} \mathrm{PFU} / \mathrm{mL}$ serum.

${ }_{\text {†PRNT }}^{80}=$ endpoint $80 \%$ neutralization titer; seroconversion is by 21 DPI. One POWV-L1- and one DTV-inoculated groundhog, each euthanized on four DPI, exhibited $>80 \%$ neutralization of POWV before inoculation.

$\ddagger$ The two POWV-L1-inoculated groundhog juveniles are not included.

determine viremia titers as $\log _{10} \mathrm{PFU} / \mathrm{mL}$ serum or homogenate (detection limits $10^{1.7}$ and 10 PFUs, respectively).

Plaque reduction neutralization test (PRNT) was performed on sera collected on 14 and $21 \mathrm{DPI}$ as previously described ${ }^{13}$ using the same virus strains as for inoculum. Postinoculation samples were serially diluted 2 -fold and tested in duplicate on Vero cells as for plaque assay to determine endpoint $80 \%$ neutralization $\left(\mathrm{PRNT}_{80}\right)$ titers.

\section{RESULTS}

Viremia profiles and tissue tropism. Low viremia titers for POWV-L1 and DTV (mean peak titers, $10^{1.7}$ and $10^{2.4} \mathrm{PFU} / \mathrm{mL}$ serum, respectively) were detected in the minority of inoculated groundhogs on three or four DPI (1/4 DTV- and 2/8 POWV-L1-inoculated). The two groundhogs with evidence of anti-POWV-L1 or DTV antibodies before inoculation (one POWV-L1- and one DTV-inoculated) did not have detectable viremia. The juvenile groundhog bled on three DPI had no detectable viremia. One of three POWV-L1-inoculated skunks had detectable viremia (mean peak titer, $10^{1.8} \mathrm{PFU} / \mathrm{mL}$ serum) on two and three DPI, and none of four DTV-inoculated skunks had detectable viremia. One of four DTV-inoculated squirrels had detectable viremia on three DPI (10 ${ }^{2.1} \mathrm{PFU} / \mathrm{mL}$ serum), and none of the three squirrels inoculated with POWV-L1 had detectable viremia (Table 1).

Isolation of DTV and POWV from tissues collected on four DPI was limited to the spleen, with minimal titers. This included DTV isolation from the spleen of $2 / 4$ squirrels $\left(10^{2.0}\right.$ and $10^{2.7}$ PFUs), 1/4 groundhogs (10 PFUs), and none of two skunks. Powassan virus-L1 was isolated from the spleen of $1 / 4$ groundhogs ( $10^{2.3}$ PFUs) and no squirrels or skunks (0/2 for each species). No viruses were isolated from the brain, heart, or kidney, and no POWV-L1 was isolated from the tissues of two inoculated juvenile groundhogs.

Serology. Before inoculation, all squirrels or skunks were seronegative for POWV-L1 and DTV. However, two groundhog adults (one inoculated with POWV-L1 and one inoculated with DTV) exhibited $>80 \%$ neutralization of POWV-L1 and DTV, respectively, before inoculation. Both of these groundhogs were euthanized on four DPI; thus, seroconversion (at 21 DPI) was not assessed. Among animals inoculated with POWV-L1 and euthanized on 21 DPI (five groundhogs [two adults, two subadults, and one juvenile], one skunk, and one squirrel), all but one groundhog adult and the squirrel seroconverted by $14 \mathrm{DPI}$. By $21 \mathrm{DPI}$, the squirrel seroconverted (PRNT 80 titer range 10-320), whereas the groundhog did not.
The juvenile groundhog inoculated with POWV-L1 and euthanized on 21 DPI seroconverted (POWV-L1 PRNT $80=40$ ). Most DTV inoculates of all three species seroconverted by 14 DPI (3/4 groundhogs, $2 / 2$ skunks, and $2 / 2$ squirrels); antibodies remained detectable on 21 DPI (PRNT 80 titer range 40-320; Table 1). There was no consistent pattern in antibody titer change in groundhogs between 14 and 21 DPI (i.e., some had up to 4-fold titer decrease, whereas others had up to 4-fold increase in titer). All skunks and squirrels increased in antibody titer between 14 and $21 \mathrm{DPI}$ (up to 8-fold increase). None of the negative (sham-inoculated) controls seroconverted by $21 \mathrm{DPI}$.

Clinical findings. No animals exhibited clinical or behavioral abnormalities during the study. All juvenile and adult groundhogs and skunks, including both negative controls and virus inoculates, maintained excellent nutritional condition throughout the study. Groundhog juveniles and squirrels, including both negative controls and virus inoculates, remained in moderate nutritional condition. Squirrel body weights remained within $1 \%$ of the pre-study weights throughout the study, and on $21 \mathrm{DPI}$, the four remaining squirrels (one control and three inoculates) had increased approximately $1 \%$ above pre-inoculation body weights. All skunks underwent a gradual weight gain throughout the study; the four skunks (one control and three inoculates) euthanized on 21 DPI had a 1.3-1.5\% overall increase in weight above pre-inoculation weights. Average internal body temperatures among inoculated skunks and squirrels decreased $0.2-0.4^{\circ} \mathrm{C}$ from two to three DPI (skunks) and two to four DPI (squirrels) and subsequently increased $1.2^{\circ} \mathrm{C}$ (skunks; up to $2.6^{\circ} \mathrm{C}$ in one individual) and $0.3^{\circ} \mathrm{C}$ (squirrels) on five DPI and returned to baseline by 7-14 DPI.

Pathology. No gross lesions were attributed to POWV-L1 or DTV infection in any animal. Gross lesions attributed to euthanasia method were observed in most individuals of all species, including negative controls, and included multiorgan congestion, primarily lungs, liver, and spleen, pulmonary edema, splenomegaly, and prominent splenic lymphoid follicles. Incidental gross findings included multifocal cestode larvae (consistent with Taenia sp.) within the hepatic parenchyma of two squirrels (both DTV inoculates) and a focal, chronic (healing) defect in the skin over the frontal bones of the skull of one DTV-inoculated squirrel. All three POWV-L1- or DTV-inoculated skunks euthanized on 21 DPI had prominent, lymph-filled lymphatic vessels along the mesenteric aspect of the small intestinal serosa.

Microscopic lesion patterns varied by species and most commonly were observed in skunks (Table 2). Six of seven inoculated skunks had inflammation in the central nervous 
TABLE 2

Summary of pathology findings in groundhogs, striped skunks, and fox squirrels experimentally inoculated with POWV-lineage 1 or DTV

\begin{tabular}{|c|c|c|c|c|c|}
\hline \multirow[b]{2}{*}{ Species } & \multirow[b]{2}{*}{ Virus } & \multirow[b]{2}{*}{ DPI } & \multicolumn{3}{|c|}{$\begin{array}{l}\text { Tissues with mild, } \\
\text { lymphoplasmacytic } \\
\text { inflammation* }\end{array}$} \\
\hline & & & Heart & Kidney & Brain \\
\hline \multirow{4}{*}{$\begin{array}{l}\text { Groundhog (Marmota } \\
\text { monax) }\end{array}$} & POWV-L1 & $4 \dagger$ & $1 / 4$ & $1 / 4$ & $1 / 4$ \\
\hline & & 21 & $0 / 4$ & $0 / 4$ & $0 / 4$ \\
\hline & DTV & $4 \dagger$ & $0 / 4$ & $0 / 4$ & $1 / 4$ \\
\hline & & 21 & $0 / 4$ & $0 / 4$ & $0 / 4$ \\
\hline \multirow{4}{*}{$\begin{array}{l}\text { Striped skunk (Mephitis } \\
\text { mephitis) }\end{array}$} & POWV-L1 & 4 & $0 / 2$ & $0 / 2$ & $2 / 2$ \\
\hline & & 21 & $0 / 1$ & $0 / 1$ & $1 / 1$ \\
\hline & DTV & 4 & $1 / 2$ & $0 / 2$ & $2 / 2$ \\
\hline & & 21 & $0 / 2$ & $0 / 2$ & $1 / 2$ \\
\hline \multirow{4}{*}{$\begin{array}{l}\text { Fox squirrel (Sciurus } \\
\text { niger) }\end{array}$} & POWV-L1 & 4 & $0 / 2$ & $0 / 2$ & $0 / 4$ \\
\hline & & 21 & $0 / 1$ & $0 / 1$ & $0 / 4$ \\
\hline & DTV & 4 & $0 / 2$ & $1 / 2$ & $0 / 4$ \\
\hline & & 21 & $0 / 2$ & $0 / 2$ & $0 / 4$ \\
\hline
\end{tabular}

DPI = days postinoculation; DTV = deer tick virus; POWV-L1 = Powassan virus-lineage 1. *Tissues examined included skeletal muscle (quadriceps and biceps), heart, trachea, lung with bronchus, thyroid gland, spleen, liver, gall bladder, kidney, adrenal gland (skunks and fox squirrels only), gonad, pancreas, esophagus, stomach, small intestine, large intestine (and cecum, if present), lymph node (mesenteric and thoracic), haired skin, adipose, bone marrow (femur), urinary bladder, eye, peripheral (sciatic) nerve, thoracic spinal cord, and brain (cerebrum, cerebellum, and brain stem). Less commonly observed lesions included lymphoplasmacytic pneumonia and thyroiditis in 1/4 POWV-L1-inoculated groundhogs on four DPI, and lymphoplasmacytic myelitis in 1/1 POWV-L1-inoculated skunks on $21 \mathrm{DPI}$ and 1/2 DTV-inoculated skunks on four DPI.

† One POWV-L1- and one DTV-inoculated groundhog, each euthanized on four DPI, exhibited $>80 \%$ neutralization of POWV before inoculation.

system. All three POWV-L1-inoculated skunks had lymphoplasmacytic encephalitis that varied from mild and focal to multifocal including perivascular (involving the meninges in $1 / 3$ ) at the early time point (four DPI; $2 / 2$ ) to moderate, perivascular, and neuroparenchymal inflammation in the cerebrum, meninges, and cervical spinal cord at the later time point (21 DPI; 1/1; Figure 1). Most (3/4) DTV-inoculated skunks (2/2 on four DPI and $1 / 2$ on $21 \mathrm{DPI}$ ) had similar, mild inflammation in the cerebrum or brain stem (Figure 2); the skunk euthanized on $21 \mathrm{DPI}$ also had focal inflammation in the meninges over the spinal cord. Non-central nervous system lesions in skunks were rare, with minimal multifocal, lymphoplasmacytic inflammation within the myocardial septum, and right and left

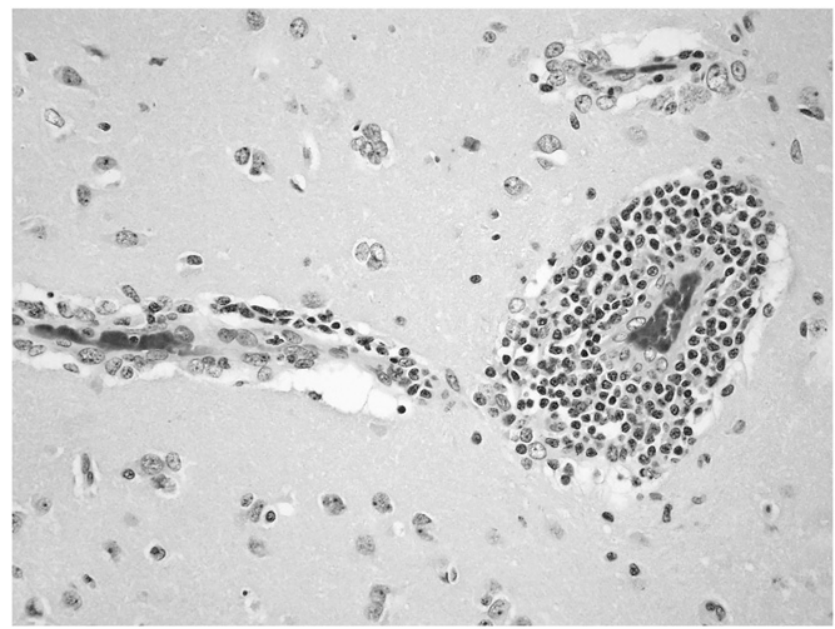

FIGURE 1. Perivascular, lymphoplasmacytic cuffs and increased numbers of astrocytes surrounding several blood vessels in the cerebrum of a striped skunk (Mephitis mephitis) inoculated with Powassan virus-lineage 1 on 21 days postinoculation. Hematoxylin and eosin $\times 40$.

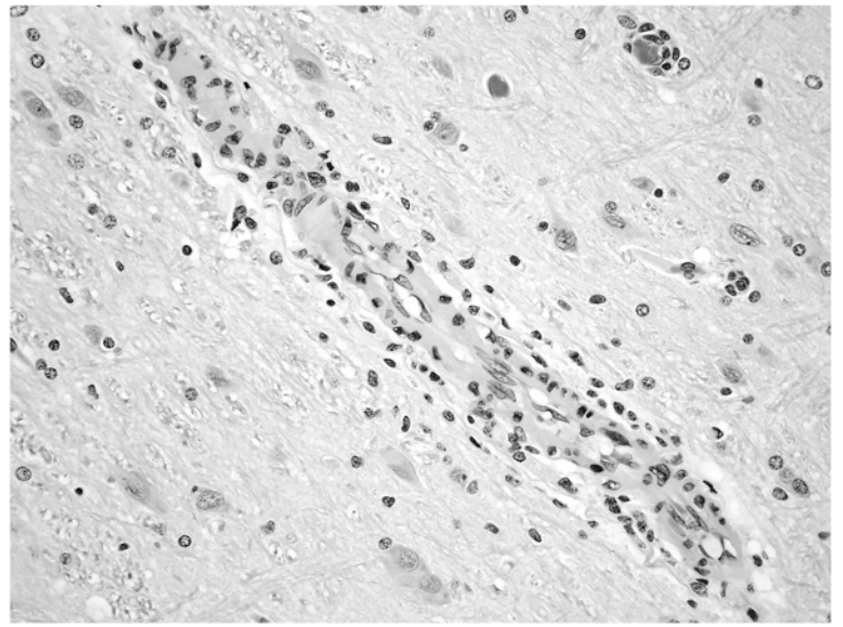

FIGURE 2. Small numbers of loosely aggregated lymphocytes and microglia surrounding a blood vessel in the brain stem of a striped skunk (Mephitis mephitis) inoculated with deer tick virus on 4 days postinoculation. Hematoxylin and eosin $\times 40$.

free walls in 1/4 DTV-inoculated skunks on four DPI. No significant microscopic lesions were observed in the shaminoculated squirrel or skunk.

In groundhogs, minimal to mild microscopic lesions were rarely observed and were primarily in those euthanized at the early time point (i.e., four DPI). Lesions consisted of lymphoplasmacytic inflammation in various organs. One of four POWV-L1-inoculated groundhogs euthanized on four DPI had mild lymphoplasmacytic, occasionally perivascular, myocarditis (in the left free wall and septum); mild, multifocal, lymphoplasmacytic, interstitial (often perivascular) pneumonia; mild, multifocal (two foci), lymphoplasmacytic, interstitial thyroiditis and nephritis; and minimal perivascular lymphoplasmacytic encephalitis in the cerebral gray matter. An additional POWV-L1-inoculated groundhog euthanized on four DPI had similar lymphoplasmacytic, interstitial nephritis occasionally associated with mineralized concretions in tubular lumens. One DTV-inoculated groundhog euthanized on four DPI had mild, cerebral, perivascular microgliosis and mild, multifocal, lymphoplasmacytic encephalitis in the cerebellar molecular layer; this was the groundhog with evidence of antiDTV antibodies before infection. None of the inoculated groundhogs euthanized on 21 DPI had microscopic lesions, except for mild, lymphoplasmacytic esophagitis in one POWVL1-inoculated groundhog. The two POWV-L1-inoculated juvenile groundhogs euthanized on three and $21 \mathrm{DPI}$ had no microscopic lesions. Microscopic lesions in fox squirrels were limited to mild, lymphoplasmacytic, interstitial nephritis in 1/4 DTV-inoculated squirrels on four DPI. Among shaminoculated groundhogs, the adult had two small foci of lymphoplasmacytic inflammation in the renal interstitium, and no significant microscopic lesions were observed in the two subadult or two juvenile groundhogs.

Incidental microscopic lesions, or those attributed to captive husbandry or euthanasia methods, included hepatic lipidosis in all groundhogs, hepatic glycogenosis, and gall bladder papillary hyperplasia in all skunks, dilated small intestinal lymphatics in all skunks euthanized on $21 \mathrm{DPI}$, and pulmonary edema and vascular congestion in most individuals of all species. Two groundhogs euthanized on $21 \mathrm{DPI}$ (one 
POWV-L1-inoculated and one DTV-inoculated) had chronic, renal infarcts. The negative control fox squirrel had suppurative nephritis suggestive of ascending bacterial infection.

\section{DISCUSSION}

The increased concern for human risk of POWV infections as well as recent delineation of DTV as a distinct POWV lineage underscores the urgent need to better understand natural transmission cycles of both currently recognized POWV lineages as well as to target high-risk regions. More information is needed on reservoir vertebrate host competence as well as diversity of competent tick vectors across changing landscapes that provide opportunities for interactions among humans, wildlife, and ticks. Detections of POWV in ticks and wildlife across a variety of geographic regions and ecosystems (e.g., California; Colorado, New York; Minnesota and British Columbia, Canada) underscore the complex and poorly understood POWV eco-epidemiology, ${ }^{1}$ which is further complicated by the dynamics of global climate change. For example, some tick species, such as $I$. scapularis, are expanding northward in latitude, bringing with it an increased infection risk of tick-borne zoonoses. ${ }^{5}$ This tick species is a known vector of DTV, among other zoonotic pathogens (e.g., Babesia microti, Borrelia burgdorferi, Borrelia miyamotoi, and Anaplasma phagocytophilum), ${ }^{14}$ and is a promiscuous and aggressive feeder that uses many wildlife and domestic animal species, as well as humans. ${ }^{1,15}$ The wide range of I. scapularis vector capabilities also is important because POWV-associated symptoms in humans may resemble those of Lyme disease, babesiosis, anaplasmosis, and B. miyamotoi disease. ${ }^{16}$ Furthermore, the isolation of POWV from Dermacentor andersoni ticks in the western United States ${ }^{17,18}$ leads to further questions of competent vector species diversity and POWV geographic distribution.

Rare POWV isolates from tissues and ticks removed from wild mammals in North America concurrent with serological evidence of POWV infections have led to established notions of major reservoir hosts. ${ }^{3,5}$ Host and tick species thought to be involved in POWV transmission cycles vary by lineage. For POWV-L1, these include mephitids (e.g., striped skunks), groundhogs, squirrels, and Ixodes cookei, and to a lesser extent rabbits and Ixodes marxi. Deer tick virus cycles between rodents (e.g., white-footed mouse, Peromyscus leucopus) and $I$. scapularis. 5,8 The present study revealed low POWV-L1 and DTV virulence in three candidate wildlife reservoir host species, the groundhog, striped skunk, and fox squirrel, following experimental infection. This determination is based on transient and low-titered viremias, minimal virus detection in tissues, lack of clinical disease, and absent to mild pathology. This pattern is consistent with previous POWV experimental studies, most of which were conducted in the 1960s-1980s and included POWV infections (lineage unknown) in a variety of wild mammal species, including Virginia opossums (Didelphis virginiana), gray foxes (Urocyon cinereoargenteus), striped skunks, groundhogs, snowshoe hares (Lepus americanus), white-footed mice, and raccoons (Procyon lotor). ${ }^{4,6,19}$ Limitations involved in past as well as the present study include small sample sizes, varied virus inoculum doses, and unknown pathogen exposure histories for some species, which should be considered in data interpretation.
Although sample sizes in the present study were small and viremia detections uncommon, two POWV-L1- and one DTVinoculated groundhog, one DTV-inoculated squirrel, and one POWV-L1-inoculated skunk became viremic, suggesting that both lineages can cause viremia in a variety of mammalian hosts. However, undetectable viremia titers most of the inoculated individuals bring into question whether there may be currently unrecognized POWV vertebrate host species that may be more susceptible to higher or longer lasting viremia titers. Data on minimum infectious POWV doses required for transmission between ticks and a variety of mammalian hosts, as well as co-feeding (i.e., tick-to-tick while concurrently feeding on the same viremic host), transmission are sparse and would greatly aid in interpreting the meaning of these data. Co-feeding is considered an important mechanism for virus transmission of some tick-borne flaviviruses, which also could include POWV-L1 and DTV. ${ }^{19,20}$ Further research is needed to confirm that viremia titers detected among numerous mammalian hosts in the present and past studies are sufficient to infect ticks. If mammals in the present study are competent hosts, then host-tick transmission of POWV-L1 or DTV may be limited to an early and short-lived time frame postinfection. ${ }^{19}$ Efficient virus transmission would seemingly be important in such a system, and POWV has proven distinctive among tick-borne pathogens in its rapid transmission from tick to host (i.e., 15 minutes), as demonstrated in laboratory mice fed upon by nymphal $I$. scapularis. ${ }^{21}$ The effects of preexisting antibodies to POWV-L1 in two, inoculated groundhogs with no detectable viremia are unknown; however, these animals were in mosquito-proof housing for $>2$ years before the study, and most of their cohorts that lacked preexisting antibodies also had no detectable viremia. Subcutaneous inoculation methods, as used in the present study to ensure delivery of known viral doses, elude aspects of natural infection such as enhanced tick saliva-activated POWV transmission, which may alter pathogenesis. ${ }^{22}$

Consistent with the absent-to-low viremia titers detected in experimentally infected groundhogs, skunks, and fox squirrels, infectious POWV-L1 and DTV were rarely detected in tissues soon after inoculation (i.e., four DPI). Furthermore, virus isolation from tissues was limited to the spleen, which as a blood-filtering organ may reflect viremic blood in the homogenized spleen sample. This finding not only suggests lack of or limited POWV-L1 and DTV replication in tissues but also supports the notion that testing of wildlife tissues likely is not an efficient method for POWV surveillance in endemic areas. $A$ recent survey of common wild mammals (primarily raccoons, skunks, and groundhogs) in presumed POWV-endemic regions of Ontario, Canada, yielded no virus isolates among tissues (including spleen) of 724 animals sampled during two transmission seasons. ${ }^{5}$ Rarely, POWV has been isolated from wild mammals, including from the blood of a red squirrel (Sciurus vulgaris; in 1962) and two groundhogs (in 1964) in the vicinity of Powassan, Ontario, as well as from pooled tissues (salivary gland, trachea, and lung) from a western spotted skunk (Spilogale gracilis) in California (in 1969), and various tissues (blood, spleen, kidney, and liver) from two groundhogs in New York (in 1964). ${ }^{17,23-25}$ Seroconversion in most of the animals tested on $21 \mathrm{DPI}$ in the present study supports use of this strategy to assess for regional POWW activity and to help determine candidate reservoir hosts. ${ }^{5,6,18,23,26}$ However, serologic distinction of POWV lineages, as well as other cross-reactive 
flaviviruses, is cumbersome via standard virus neutralization assay. ${ }^{12}$ Serology remains the most common method to diagnose POWV infections in humans, and recent developments (e.g., indirect enzyme immunoassay test panel) allow for improved detection capabilities ${ }^{27}$ that potentially could be applied to wildlife samples.

Pathology attributable to POWV-L1 or DTV infection in striped skunks, fox squirrels, and groundhogs generally was absent to mild, with subtle species differences. In all species, microscopic evidence of POWV infections was limited-tomild, lymphoplasmacytic inflammation, which was most commonly in the cerebrum, and to a lesser extent in the cerebellum and cranial spinal cord. Histology was assessed at early (four DPI) and late time points (21 DPI), with lesions limited to the earlier time point in groundhogs and fox squirrels. However, one DTV- and one POWV-L1-inoculated skunk examined on 21 DPI had inflammation in the cerebrum and meninges, as well as in the spinal cord in the POWV-L1inoculated individual. These central nervous system lesions were more severe on $21 \mathrm{DPI}$ than those observed in all three species at the earlier time point (four DPI). Thus, in groundhogs and squirrels, mild inflammation in the central nervous system may be temporally limited to soon after infection with rapid resolution, whereas in skunks, lesion progression was evident in some individuals between four and $21 \mathrm{DPI}$. Prior research suggests that the duration of resolution of POWV-induced encephalitic lesions in hosts varies by species. For example, in contrast to fox squirrels and groundhogs, white-footed mice intracerebrally inoculated with POWV-developed lymphocytic encephalitis from 5 to $15 \mathrm{DPI}$ that resolved by $28 \mathrm{DPI} .^{19}$ Lesions observed in the present study were similar, albeit less severe, to those described in most of the intravenously inoculated rabbits, intracerebrally inoculated white-footed mice, and several intravenously and intracerebrally inoculated horses. The mice and rabbits lacked clinical signs, and no virus was detected in tissues by virus isolation in horses and PCR in mice. ${ }^{19,28}$ This inflammation induced by POWV would likely be indistinguishable from that of other flaviviruses (e.g., West Nile virus) in these mammals. ${ }^{29}$ All inoculated and control animals were in apparent good health and maintained nutritional condition and body weight throughout the study, and the described microscopic lesion pattern in the central nervous system would not be expected to compromise survival in immunocompetent hosts. ${ }^{19} \mathrm{Al}-$ though a definitive association between POWV infections and lesions such as inflammation cannot be made without immunohistochemistry or in situ hybridization, the temporal and species patterns and lack of similar lesions in negative control animals in the present study support a causative association. Other viruses that can cause encephalitis in these species (e.g., canine distemper and rabies viruses) are considered unlikely, as they had no access to the outdoors or other animals while in captivity, and were either captive born and reared (skunks), from a long-standing captive colony (groundhogs), or apparently healthy on capture and throughout the subsequent study period (squirrels).

Recent surveillance among potential POWV-amplifying wild mammalian hosts in Ontario, Canada, ${ }^{5,30}$ concurrent with results from the present study, suggests that a better understanding of this and other aspects of POWV ecoepidemiology in North America is needed, especially given increased numbers of reported POWV and DTV human cases, as well as predictions that these numbers may continue to increase with climate change. ${ }^{31}$ The diversity of candidate or presumed mammalian reservoir hosts of POWV, evident in the present study and supported by past findings, ${ }^{1}$ underscores this need. The recent focus on the public health threat posed by the DTV lineage of POWV is in part due to its primary recognized vector, I. scapularis, and its tendency to aggressively feed on a wide variety of hosts. ${ }^{1}$ Thus, additional research on tick feeding and wildlife host dynamics is needed to better understand these relationships and natural transmission dynamics that support virus maintenance and lead to human infections.

Received February 7, 2020. Accepted for publication August 13, 2020. Published online January 18, 2021.

Acknowledgments: We are grateful to Greg Ebel at Colorado State University for providing virus isolates used in this study and Paul Oesterle for assistance in manuscript preparation.

Financial support: This research was funded by the Natural Sciences and Engineering Research Council of Canada 2015-04088, Colorado State University Animal Modules Core 21-21500, and the U.S. Department of Agriculture, Animal, and Plant Health Inspection Service.

Authors' addresses: Nicole M. Nemeth, Department of Population Health, Southeastern Cooperative Wildlife Disease Study, University of Georgia, Athens, GA, and Department of Pathology, Southeastern Cooperative Wildlife Disease Study, University of Georgia, Athens, GA, E-mail: nmnemeth@uga.edu. J. Jeffrey Root, United States Department of Agriculture/APHIS, National Wildlife Research Center, Fort Collins, CO, E-mail: jeff.root@usda.gov. Airn E. Hartwig, Richard A. Bowen, and Angela M. Bosco-Lauth, Department of Biomedical Sciences, College of Veterinary Medicine and Biomedical Sciences, Colorado State University, Fort Collins, CO, E-mails: airn.tolnay@colotate.edu, rbowen@ rams.colostate.edu, and mopargal@rams.colostate.edu.

\section{REFERENCES}

1. Hermance ME, Thangamani S, 2017. Powassan virus: an emerging arbovirus of public health concern in North America. Vector Borne Zoonotic Dis 17: 453-462.

2. Hinten SR et al., 2008. Increased recognition of Powassan encephalitis in the United States, 1999-2005. Vector Borne Zoonotic Dis 8: 733-740.

3. Artsob H, Spence L, Surgeoner G, McCreadie J, Thorsen J, Thng C, Lampotang V, 1984. Isolation of Francisella tularensis and Powassan virus from ticks (Acari, Ixodidae) in Ontario, Canada. J Med Entomol 21: 165-168.

4. Kokernot RH, Radivoje B, Anderson RJ, 1969. Susceptibility of wild and domestic mammals to four arboviruses. Am J Vet Res 30: 2197-2203.

5. Smith K, Oesterle PT, Jardine CM, Dibernardo A, Huynh C, Lindsay R, Pearl DL, Bosco-Lauth AM, Nemeth NM, 2018. Powassan virus and other arthropod-borne viruses in wildlife and ticks in Ontario, Canada. Am J Trop Med Hyg 99: 458-465.

6. Zarnke RL, Yuill TM, 1981. Powassan virus infection in snowshoe hares (Lepus americanus). J Wildl Dis 17: 303-310.

7. Corrin T, Greig J, Harding S, Young I, Mascarenhas M, Waddell $L A, 2018$. Powassan virus, a scoping review of the global evidence. Zoonoses Public Health 65: 595-624.

8. Ebel GD, 2010. Update on Powassan virus: emergence of a North American tick-borne flavivirus. Annu Rev Entomol 55: 95-110.

9. El Khoury MY et al., 2013. Potential role of deer tick virus in Powassan encephalitis cases in lyme disease-endemic areas of New York, USA. Emerg Infect Dis 19: 1926-1933.

10. Dupuis AP, Peters RJ, Prusinski MA, Falco RC, Ostfeld RS, Kramer LD, 2013. Isolation of deer tick virus (Powassan virus, lineage II) from Ixodes scapularis and detection of antibody in vertebrate hosts sampled in the Hudson Valley, New York state. Parasit Vectors 6: 185.

11. Nemeth NM, Bosco-Lauth AM, Williams LM, Bowen RA, Brown JD, 2017. West Nile virus infection in ruffed grouse (Bonasa 
umbellus): experimental infection and protective effects of vaccination. Vet Pathol 54: 901-911.

12. Beaty BJ, Calisher CH, Shope RE, 1995. Diagnostic procedures for viral, rickettsial, and chlamydial infections. Lennette $\mathrm{EH}$, Lennette DA, Lennette ET, eds. Arboviruses. Washington, DC: American Public Health Association, 189-212.

13. Nofchissey RA et al., 2013. Seroprevalence of Powassan virus in New England deer, 1979-2010. Am J Trop Med Hyg 88: 1159-1162.

14. Eisen L, 2018. Pathogen transmission in relation to duration of attachment by Ixodes scapularis ticks. Ticks Tick Borne Dis 9: 535-542.

15. Bouchard C, Leonard E, Koffi JK, Pelcat Y, Peregrine A, Chilton N, Rochon K, Lysyk T, Lindsay LR, Ogden NH, 2015. The increasing risk of lyme disease in Canada. Can Vet J 56: 693-699.

16. Caulfield AJ, Pritt BS, 2015. Lyme disease coinfections in the United States. Clin Lab Med 35: 827-846.

17. Whitney $E$, Jamnback $H, 1965$. The first isolations of Powassan virus in New York state. Proc Soc Exp Biol Med 119: 432-435.

18. Thomas LA, Kennedy RC, Eklund CM, 1960. Isolation of a virus closely related to Powassan virus from Dermacentor andersoni collected along North Cashe La Poudre river, Colo. Proc Soc Exp Biol Med 104: 355-359.

19. Mlera L, Meade-White K, Saturday G, Scott D, Bloom ME, 2017. Modeling Powassan virus infection in Peromyscus leucopus, a natural host. PLoS Negl Trop Dis 11: 1-19.

20. Havlíková S, Ličková M, Klempa B, 2013. Non-viraemic transmission of tick-borne viruses. Acta Virol 57: 123-129.

21. Ebel GD, Kramer LD, 2004. Short report: duration of tick attachment required for transmission of Powassan virus by deer ticks. Am J Trop Med Hyg 71: 268-271.
22. Hermance ME, Thangamani S, 2015. Tick saliva enhances Powassan virus transmission to the host, influencing its dissemination and the course of disease. J Virol 89: 7852-7860.

23. Johnson $\mathrm{HN}, 1987$. Isolation of Powassan virus from a spotted skunk in California. J Wildl Dis 23: 152-153.

24. McLean DM, Best JM, Mahalingham S, Chernesky MA, Wilson WE, 1964. Powassan virus: summer infection cycle, 1964. Can Med Assoc J 91: 1360-1362.

25. McLean DM, Larke RP, 1963. Powassan and Silverwater viruses: ecology of two Ontario arboviruses. Can Med Assoc J 88: 182-185.

26. McLean DM, Ladyman SR, Purvin-Good KW, 1968. Westward extension of Powassan virus prevalence. Can Med Assoc J 98: 946-949.

27. Thomm AM, Schotthoefer AM, Dupuis AP, 2nd, Kramer LD, Frost HM, Fritsche TR, Harrington YA, Knox KK, Kehl SC, 2018. Development and validation of a serologic test panel for detection of Powassan virus infection in U.S. patients residing in regions where lyme disease is endemic. mSphere 3: e00467-17.

28. Little PB, Thorsen J, Moore W, Weninger N, 1985. Powassan virus encephalitis - a review and experimental studies in the horse and rabbit. Vet Pathol 22: 500-507.

29. Root JJ, Oesterle PT, Nemeth NM, Klenk K, Gould DH, McLean RG, Clark L, Hall JS, 2006. Experimental infection of fox squirrels (Sciurus niger) with West Nile virus. Am J Trop Med Hyg 75: 697-701.

30. Smith KA, Oesterle PT, Jardine CM, Dibernardo A, Huynh C, Lindsay R, Pearl DL, Nemeth NM, 2019. Tick infestations of wildlife and companion animals in Ontario, Canada, with detection of human pathogens in Ixodes scapularis ticks. Ticks Tick Borne Dis 10: 72-76.

31. Fatmi SS, Zehra R, Carpenter DO, 2017. Powassan virus - a new reemerging tick-borne disease. Front Public Health 5: 342. 